Luyando Cuevas, J. R. (2013). Desigualdad salarial: antes y durante la apertura comercial mexicana. Revista Lebret (5). Bucaramanga, Colombia: Universidad Santo Tomás, pp. 29-50

\title{
Desigualdad salarial antes y durante la apertura comercial mexicana"
}

\author{
Wage inequality: before and during \\ the Mexican trade liberalization
}

José Raúl Luyando Cuevas ${ }^{l}$

\section{Resumen}

Basados en la propuesta teórica sobre la existencia de una relación positiva entre el nivel de escolaridad y el nivel salarial, se realiza un estudio sobre la desigualdad salarial en México por nivel escolar. Se consideró como año referente 1984 (antes de la apertura comercial de México) y se comparó con los años 1992, 2000 y 2006, en los que México se encontraba en diferentes etapas de su apertura comercial.

\section{Palabras clave}

Desigualdad, salario, Escolaridad, Apertura, Productividad.

\section{Códigos de Clasificación JEL: I24, J24, J31}

\begin{abstract}
Based on the theoretical proposal of the existence of a positive relationship between the education level and the salary level, was made this study about wage inequality in Mexico by school level. 1984 was considered as reference year (before the commercial opening of Mexico) and it was compared with years 1992, 2000 and 2006, when Mexico was in different stages of the trade liberalization process.
\end{abstract}

\section{Keywords}

Inequality, Salary, Schooling, Openness, Productivity.

* $\quad$ Este artículo de investigación se desarrolla a partir de un proyecto titulado: Factores que han influido en la determinación de la tasa salarial en el mercado de trabajo mexicano en tres periodos 1984, 1992 y 2000. La investigación fue financiada por CONACYT México.

1 Postdoctor de la Universidad Autónoma Metropolitana, México. Profesor de tiempo completo en el Instituto de Investigaciones Sociales de la Universidad Autónoma de Nuevo León, México. Correo electrónico: jrlc9@ hotmail.com 


\section{Introducción}

La desigualdad salarial es un tema de gran relevancia en toda economía y es uno de los más estudiados en el mundo². En el caso de México, el trabajo de Castro y Huesca (2007) hace una revisión exhaustiva de las principales investigaciones que se han realizado sobre México a este respecto. En una primera aproximación, estos autores señalan que una parte de las investigaciones encuentran que:

[...] puede afirmarse que el incremento de la desigualdad salarial mostrado a partir de la segunda mitad de la década de 1980 y hasta mediados de la década de 1990, así como su posterior reducción, tienen como causante principal los cambios relativos en los retornos a la educación o calificación laboral. De lo anterior surge una pregunta ¿cuál ha sido el comportamiento de la disparidad salarial al interior de los grupos educativos o sectores? (p. 242)

Para posteriormente resaltar que:

La disparidad salarial se incrementó no sólo entre diferentes grupos educativos, sino al interior de los mismos hasta la segunda mitad de la década de 1990 , aunque no parece existir un comportamiento claro sobre la desigualdad al interior de los grupos educativos a partir de la segunda mitad de la década de 1990 (Castro y Huesca, 2007, p. 243).

Además de lo anterior, otras investigaciones sustentan que la desigualdad salarial también puede ser explicada por el efecto que tuvo la apertura comercial al interior del mercado laboral mexicano, principalmente en lo referente a lo regional y tecnológico. Una gran cantidad de las investigaciones, ponen especial atención al periodo donde se inicia y expande la liberalización comercial en México. Autores como Stiglitz (2002)3 , Feliciano (2001), Hanson y Harrison (1995 y 1999), Cragg y Epelbaum (1996) y Hanson (2003 y 2005), encuentran en general, que la dispersión salarial en México se incrementó debido a la política de apertura comercial llevada a cabo por el gobierno. De hecho, Stiglitz (2002, p.88) deja entrever que el gobierno mexicano realizó una apertura comercial apresurada, en el sentido de que no hubo una planeación estratégica pensada para beneficiar al total de la economía, y por tanto, a la mayoría de la población ${ }^{4}$.

2 En los años setentas y ochentas uno de los principales argumentos para diagnosticar la desigualdad salarial que padecían países como los Estados Unidos de Norteamérica, era que los trabajadores con mayores skills -muy relacionadas con los niveles y calidad de la educación obtenida- conseguían salarios mayores en perjuicio de los trabajadores menos calificados o con menores skills que cada vez obtenían salarios más bajos, Murphy y Whelch (1991); Blackburn, Bloom y Freeman (1990/91); Kosters (1990); Juhn, Murphy y Pierce (1993), entre otros. Aunque también se daban argumentos, que de forma indirecta están relacionados a lo anterior, como la apertura comercial creciente, el crecimiento poblacional y el desarrollo tecnológico, Slaughter (1999); Murphy y Welch (1991); Bluestone y Harrison (1988); Davis y Haltiwanger (1991); Lawrence y Slaughter (1993); Berman, Bound y Griliches, (1994); Krueger (1993), entre otros.

3 "Incluso en países que lograron un cierto crecimiento como México, los beneficios fueron acaparados por el 30 por ciento y especialmente por el 10\% más rico. Los pobres apenas ganaron y muchos están peor". (Stiglitz, 2002, p.118).

4 Muchas pequeñas y medianas empresas quebraron, rompiéndose múltiples cadenas productivas que dejaron en el desempleo a una gran cantidad de trabajadores con habilidades propias del sistema de producción por sustitución de importaciones. Habilidades que ya no eran aptas para los nuevos procesos productivos. 
En lo referente a lo regional, en general, se argumenta que los Estados más beneficiados en México fueron los del norte, principalmente los que colindan con los Estados Unidos de Norteamérica, Rodríguez y Sánchez (2002), Chiquiar (2004) y Castro y Huesca (2005). En el otro caso, en general, se plantea que con la apertura se aceleró el cambio tecnológico, con lo que se afectó la demanda laboral, sesgándose en cierta medida hacia los trabajadores con mayores grados escolares, Hanson y Harrison (1995), Cragg y Epelbaum (1996), Meza (1999) y López (2001 y 2004). Por último, otras investigaciones sostienen que son factores institucionales -más allá de la apertura comercial y la escolaridad- los que están afectando el mecanismo de distribución, es decir, la actuación de los sindicatos y la intervención gubernamental en las negociaciones salariales (para contener los incrementos) o para fijar salarios mínimos, Popli, Gurleen (2005 y 2006) y Herrera y Melgoza (2003).

Con excepción quizá de los argumentos institucionales, los otros, en última instancia, en casi todos los casos, también consideran como explicación los retornos a la educación o calificación de los trabajadores. Con respecto a la apertura comercial, cuando no es utilizado como argumento principal, lo esgrimen como un parámetro.

Dado lo anterior, la presente investigación estudia la desigualdad salarial no sólo entre diferentes grupos educativos, sino al interior de los mismos en cuatro años que creemos claves y que nos pueden dar indicios acerca de alguna tendencia. El año 1984 proporciona un panorama de las características de los trabajadores y del mercado laboral antes de que se diera la apertura comercial; el año de 1992 nos da la posibilidad de ver cómo cambiaron esas características cuando México ingresa al Acuerdo General sobre Aranceles Aduaneros y Comercio (GATT, por sus siglas en inglés) y antes de que entrara en vigor el Tratado de Libre Comercio de América del Norte (NAFTA, por sus siglas en inglés); el año 2000 nos da la posibilidad de ver cómo cambiaron esas características, pues es un año en el que ya se habían consolidado muchos de los efectos de la entrada de México al GATT, y se podían apreciar también los efectos del NAFTA. Por último, el año 2006 nos da un panorama de una etapa de consolidación de los acuerdos de libre comercio, pues nos encontramos a veintiún años del ingreso de México al GATT y a doce años del ingreso al NAFTA ${ }^{5}$.

Antes del año 1980, los esfuerzos de los gobiernos mexicanos estaban enfocados en el llamado modelo de sustitución de importaciones. Pero a partir de 1983, el gobierno mexicano inicia algunos programas orientados a facilitar la movilidad de la mano de obra y el ajuste del mercado laboral. En 1985 México ingresa al GATT, donde el gobierno mexicano se compromete a rebajar los aranceles y a eliminar barreras

5 México ha suscrito varios acuerdos de libre comercio con diferentes países, pero el GATT y NAFTA han sido los más significativos. El primero por ser el que inicia la apertura de la economía mexicana y el segundo porque se hace con el país con la economía más poderosa del planeta y a donde México destina alrededor del $90 \%$ de sus exportaciones. 
no arancelarias ${ }^{6}$, terminando así con cuatro décadas de industrialización mediante el modelo de sustitución de importaciones. En 1989 el gobierno mexicano modera las restricciones para extranjeros sobre posesión de activos en territorio nacional ${ }^{7}$. El primero de enero de 1994 entra en vigor el NAFTA, y con él, se consolidan y extienden las reformas acontecidas en 1985 y 1989.

La investigación así planteada se realiza con herramientas de análisis convencional, pero los resultados que se obtienen son interesantes y dan un panorama particular de lo que está aconteciendo con la relación escolaridad-salario, contribuyendo de esta manera a la discusión que sobre este tema se ha dado a nivel mundial.

\section{La Desigualdad inevitable y el capital humano}

Desde algunas perspectivas sociales se concibe a la desigualdad salarial como un problema que afecta el bienestar de la población y que se debe erradicar buscando la equidad. Pero en un sistema económico capitalista, como el que existe en la gran mayoría de los países del mundo, la desigualdad juega un papel importante. Pues como lo señala Welch (1999) la desigualdad que es aceptada por la sociedad, es una que genera competencia y oportunidades al interior de todo mercado laboral ${ }^{8}$.

Los salarios tienen el papel de distribuir parte de la riqueza generada por una economía, y si ésta se hiciera de forma eficiente, como lo predice la teoría de la competencia perfecta, se podría decir que cada asalariado obtendría el salario que se merece. En este caso, las diferencias salariales serían aceptadas por la sociedad, en el entendido de que los salarios estarían atados al producto generando por cada uno de los trabajadores, pues se supone que el mercado en la búsqueda de la eficiencia económica es imparcial en sus mecanismos de distribución. Algo que estaría asociado a los retornos a la educación o las habilidades que obtiene el trabajador a lo largo de su vida, que en inglés se denomina "skills", una palabra que tiene varias acepciones como: habilidad, destreza, especialización, capacitación, responsabilidad y experiencia. La calidad y cantidad de "skills" con que cuenta cada trabajador y que redundan en su productividad, y por tanto, en el salario que obtienen, tienen una

6 El gobierno mexicano protegió, durante décadas, a los artículos producidos al interior de su economía mediante el uso de tarifas y permisos de importación. Las tarifas incrementaban el precio de importación de los artículos, mientras que los permisos limitaban el número de artículos importados.

7 "Como es del dominio público, el gobierno de De la Madrid ha enfatizado en varias ocasiones su deseo de fomentar la llegada de nueva inversión extranjera directa ofreciendo aplicar con mucha flexibilidad, en un tratamiento caso por caso, las leyes mexicanas restrictivas sobre inversión extranjera y transferencia de tecnología expedidas en el sexenio de Echeverría. En este sentido, a inicios de 1984 el gobierno expidió nuevos lineamientos que especifican las condiciones en las cuales se puede autorizar la propiedad extranjera mayoritaria tanto en empresas nuevas como en las que ya están operando. A este respecto, se considera la posibilidad de otorgar exenciones en industrias que sustituyan importaciones clave, en industrias con alto potencial de exportación, en actividades intensivas en mano de obra y en algunas otras actividades específicas" (Guillén, 1988, p.54).

8 "Without inequality of priorities and capabilities, there would be no trade, no specialization, and no surpluses produced by cooperation. Incidentally, there would be no economics, and we would all be selling insurance!" (Welch, 1999, p.2) 
gran cantidad de orígenes, pero en las economías capitalistas uno de los factores que mayormente la afectan, y en lo cual hay un consenso teórico y empírico, es el nivel escolar adquirido. En este sentido, como ya señalamos, la desigualdad puede verse como un bien económico que genera oportunidades, pues las diferencias salariales producidas por escasez o abundancia de trabajadores con determinado perfil de "skills", creará incentivos para que las nuevas generaciones busquen capacitarse de la mejor manera y puedan obtener los puestos laborales más valorados por el mercado. Es decir, un incremento en la desigualdad asociada con el aumento de los retornos a la educación, es desde la perspectiva de Welch (1999), un problema a corto plazo para la población con escolaridad inferior a la media. Pero a más largo plazo, el aumento en el rendimiento a la educación, lo que estría creando son oportunidades para los futuros trabajadores y por consecuencia para un mayor bienestar de la población.

Por tanto, la libertad de elegir cuando la teoría del mercado laboral se cumple, generaría movilidad social entre todos aquellos deseosos de obtener una mayor capacitación a cualquier nivel, y con ello, tener una mejor expectativa de vida. Es decir, en términos generales, se podría decir que los individuos, bajo los supuestos antes señalados, tienen la facultad de incidir en sus vidas -dadas sus prioridades y capacidades- mediante su esfuerzo en la adquisición de "skills", y por este conducto, en el tipo de empleo y salario que podrán conseguir.

El problema con lo antes señalado, es que es difícil que se cumplan todas las condiciones que impone la teoría de mercado para funcionar de esa manera, por lo que en general, lo que existe en nuestras economías son mercados imperfectos. Es decir, existen otros factores que afectan el mecanismo de distribución, como pueden ser los sindicatos o la intervención gubernamental (generalmente para fijar salarios mínimos $^{9}$ en busca de la equidad social). Estas intervenciones vuelven menos nítido el mecanismo teórico, pues en ellas entra en juego la condición humana, con todas sus virtudes y defectos -en la teoría de mercado suponemos un ente metafísico denominado mercado, que por lo mismo se supone imparcial-.

No obstante, cuando la condición humana interviene, pueden existir vicios tan nefastos como la corrupción o el nepotismo, los cuales pueden accionar mecanismos arbitrarios y hasta ilegales de distribución. La permeabilidad de estos vicios a todos los niveles de la sociedad o una mala intervención gubernamental pueden generar desigualdades que ya no son aceptables -más cuando las diferencias salariales se tornan extremas- pues esta situación es interpretada como injusta, y en ese sentido, se vuelve un factor que destruye el tejido social, por lo que la desigualdad ya no será vista como un bien que genera oportunidades. El problema con lo anterior, es que el

9 En el caso de México, se supone un salario mínimo que sea suficiente para satisfacer las necesidades normales de un jefe de familia, en el orden material, social y cultural, y para proveer a la educación obligatoria de los hijos Definición de salario mínimo en el artículo 123 de la Constitución Política de los Estados Unidos Mexicanos. Consultado en: http://info4.juridicas.unam.mx/ijure/fed/9/124.htm 
ideal de la movilidad social basado en el esfuerzo individual y la libertad de elegir se ve influido por factores humanos. Los individuos ya no tendrán certidumbre sobre sus esfuerzos anteriores (escolares) y actuales (en el lugar de trabajo), pues en la valoración de estos podrían entrar cuestiones o atributos que ya no tienen que ver con su productividad, por tanto, no tendrán certeza sobre el peso de su esfuerzo individual para afectar sus condiciones laborales (principalmente su nivel salarial).

Pero aun existiendo otros factores que influyen en el nivel salarial de los trabajadores, uno de los principales atributos o señales que considera la demanda laboral para contratar a un trabajador, en un determinado puesto u ocupación, es su nivel escolar, pues en términos generales, es un indicio de los conocimientos que ha adquirido y quizá de las habilidades con que cuenta el trabajador. En este sentido, el nivel y la calidad de la educación adquirida, serán un signo tomado en cuenta por las empresas para contratar trabajadores, pues supondrían que afecta la productividad del trabajador, y mediante esta última, el salario del trabajador y las ganancias de la empresa, además de que la escolaridad es un distintivo que el empleador puede compulsar fácilmente mediante un documento oficial que es aceptado socialmente. De este modo, la desigualdad salarial, en un principio, se puede analizar desde la relación escolaridad-salario. Pero la debilidad de esta relación o su fortaleza también nos podrán indicar la magnitud de la influencia que tienen otros factores.

\section{Características de las bases de datos y de las variables utilizadas}

Para realizar el análisis se utilizaron las Encuestas Nacionales de Ingresos y Gastos de los Hogares (ENIGH) de los años 1984, 1992, 2000 y 2006, elaboradas en México por el Instituto Nacional de Estadística y Geografía (INEGI). Con el propósito de crear las bases de datos que se necesitaban para cada año, se utilizaron sólo dos de las bases internas que se encuentran en las ENIGH. La de Ingresos, que detalla el monto de los ingresos de los trabajadores y de dónde provienen, y la llamada Person, donde se detallan las características escolares, sociales y laborales de los miembros del hogar. Estas dos bases se fusionaron en una sola mediante dos campos comunes, el campo llamado Folio, el cual es un identificador único del hogar, y el campo Numrem, el cual señala el número de renglón del residente del $\operatorname{hogar}^{10}$. La intención al fusionarlas era tener identificadas a las personas que, dentro de la familia, obtenían algún ingreso, saber cuál era el monto del ingreso, de dónde provenía y cuáles eran las características sociales y laborales de estas personas.

10 Para realizar la fusión se utilizó el programa Acces. Cabe señalar que para los años 1984, 2000 y 2006 no hubo mayor problema, pero para el año 1992 tuvimos que superar varios de ellos. En primer lugar, hay una buena cantidad de trabajadores que no tenían el campo Numrem; después de intentar varias soluciones se decidió dejarlos fuera por considerar que era la solución más indicada. En segundo lugar, varios campos estaban aglutinados en uno solo, por lo que se procedió a dividirlos por medio de la longitud de cada uno de los campos aglutinados; esto se realizó con la programación del mismo Acces. 
En los cuatro años utilizamos como salario lo que se denomina en las encuestas "ingreso mes pasado", es decir, el último ingreso que percibió el trabajador. En específico, hablamos de los sueldos, salarios o jornal y horas extras, considerando sólo los ingresos netos por remuneraciones al salario, en otras palabras, las percepciones en efectivo que obtienen los miembros del hogar a cambio de la venta de su fuerza de trabajo a una empresa, institución o patrón con quien han establecido determinadas condiciones de trabajo mediante un contrato o acuerdo ${ }^{11}$. En el caso de la educación formal o escolaridad, se tuvo que hacer una unificación de criterios, ya que las pautas para codificar los niveles escolares en las cuatro encuestas fueron diferentes. Por tanto, la codificación utilizada fue la siguiente: 0 para trabajadores sin instrucción; 3 para trabajadores con primaria incompleta; 6 para trabajadores con primaria completa; 8 para trabajadores con secundaria incompleta; 9 para trabajadores con secundaria completa; 11 para trabajadores con pre-universidad incompleta; 12 para trabajadores con pre-universidad completa; 15 para trabajadores con superior incompleta; 17 para trabajadores con superior completa y 18 para trabajadores con estudios de posgrado.

\section{Niveles salariales en México}

En este apartado, para tener un panorama general de lo que ocurría con los salarios en los cuatro años, se hace un análisis de ellos teniendo como parámetro el salario mínimo $^{12}$. Se comienza por señalar que el salario mínimo nominal mensual en 1984 fue de 24,480.00 (veinticuatro mil cuatrocientos ochenta pesos); para el año de 1992 de 399,900.00 (trescientos noventa y nueve mil novecientos pesos); para el año 2000 de 1,137.00 (mil ciento treinta y siete nuevos pesos) y para el año 2006 de 1,460.00 (mil cuatrocientos sesenta pesos) (Aguirre, 1993) (13 $^{3}$. Para tener una mejor manera de analizar los datos, también obtenemos el salario promedio por año. Y obtenemos que para 1984, el salario promedio fue de 28,115.28 (veintiocho mil ciento quince pesos, con veintiocho centavos); en 1992, fue de 850,159.21 (ochocientos cincuenta mil ciento cincuenta y nueve pesos, con veintiún centavos); en el año 2000, fue de $2,892.80$ (dos mil ochocientos noventa y dos pesos, con ochenta centavos) y en el año 2006, fue de 5,617.96 (cinco mil seiscientos diez y siete pesos, con noventa y seis centavos). En los cuatro años, el salario promedio siempre es mayor al salario mínimo. Pero cabe resaltar que aunque en 1984 el salario promedio es mayor al mínimo, lo es por muy poco. A partir de 1992, el salario promedio se aleja cada vez y en mayor medida del mínimo, lo que se podría ver como un buen augurio para los trabajadores asalariados. Pero para tener un panorama más completo, debemos hurgar un poco

11 Cuando el trabajador indicaba que tenía más de un ingreso por este mismo rubro, se sumaron estos ingresos y se tomaron como uno solo, para no tener duplicidad de trabajadores. Es decir, si identificábamos, por medio del número de renglón del residente del hogar, a alguna persona con dos ingresos o más, acumulábamos esos ingresos, en el sentido de no crear distorsiones en el interior de la muestra, esto se realizó por medio del programa Crystal Reports.

12 Que sigue siendo un referente para las negociaciones salariales en México.

13 Para encontrar el salario mínimo mensual, se multiplicó el salario mínimo diario por 30. 
más. Un dato que esclarece más, sería la cantidad de trabajadores que ganaban el salario mínimo en los diferentes años: en 1984, el 52.4\% de los trabajadores ganaban un salario mínimo o menos y el 88.9\% dos salarios mínimos o menos; para 1992, el $29.3 \%$ de los trabajadores ganaban un salario mínimo o menos y el $68.5 \%$ dos salarios mínimos o menos; para el año 2000, el 19.3\% de los trabajadores ganaba un salario mínimo o menos y $55.3 \%$ dos salarios mínimos o menos; por último, para el año 2006, el $12.1 \%$ de los trabajadores ganaba un salario mínimo o menos y $37.8 \%$ dos salarios mínimos o menos. El porcentaje de trabajadores que ganaban un salario mínimo o menos fue decreciendo en la apertura comercial, lo que de nueva cuenta parecería una buena noticia para los trabajadores mexicanos, pues sin tomar en cuenta otras circunstancias, se podría decir que en la apertura comercial una mayor proporción de trabajadores ganaban más salarios mínimos.

El problema con el anterior análisis, es que no toma en cuenta el panorama completo, pues estamos hablando de salarios nominales, no de salarios reales, que es lo que verdaderamente importa a todo trabajador (su poder adquisitivo). La Figura 1 muestra el salario mínimo real diario promedio nacional para los años de estudio.

Figura 1. Salario Mínimo Real Promedio Nacional (pesos de 1994)

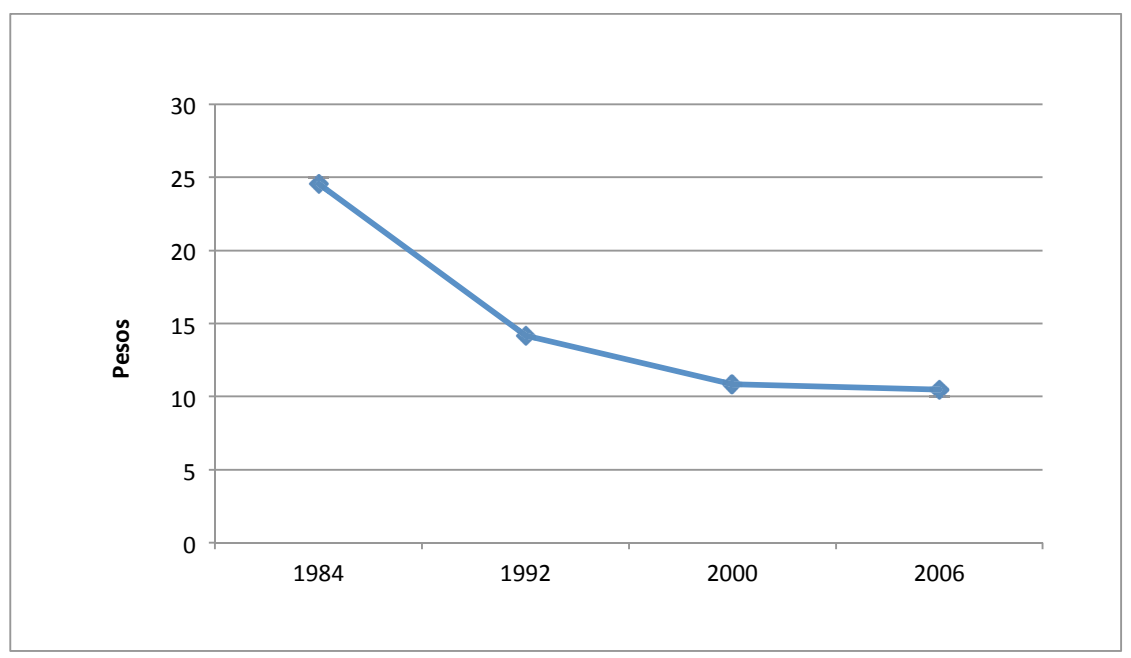

Fuente: Autor con datos de la Comisión Nacional de Salarios Mínimos, Secretaría del Trabajo y Previsión Social (aquí cabe recordar, que en el año de 1993 se le quitan al peso mexicano tres ceros, lo que en una primera instancia se conocieron como nuevos pesos).

En la Figura 1 se puede observar que el salario mínimo real diario a precios de 1994, en 1984 era de 24.56 (pesos mexicanos), en 1992 de 14.18 pesos, para el 
año 2000 de 10.85 pesos y para el año 2006 de 10.48 pesos. El salario mínimo real tiene una tendencia claramente decreciente. En este sentido, se puede calcular que el salario real de 1992 representaba el $57.74 \%$ del de 1984, el salario real del año 2000 representaba el $44.18 \%$ del de 1984 y el salario real del año 2006, representaba el 42.67\%. Considerando esta situación, dos salarios mínimos del año 2000, representaban menos de un salario mínimo de 1984.

Para el año de 1984, el 52.4\% de los trabajadores ganaban un salario mínimo o menos, y para el año 2000 tenemos que los trabajadores que ganaron dos salarios mínimos o menos eran 55.3\%, pero estos dos salarios mínimos del año 2000 representan menos de un salario mínimo de 1984. Por tanto, hay un mayor porcentaje de trabajadores que en el año 2000, ganan un salario mínimo real de 1984. Para el año 2006 la caída en el poder adquisitivo ya no es tan marcada, y de hecho, con los datos que tenemos hay alguna recuperación. Para este año, dos salarios mínimos representan alrededor del 85\% de un salario mínimo en 1984. Pero en el año 2006, tan sólo el 37.8\% de los trabajadores ganaban dos salarios mínimos. Por aproximación, se pude decir que alrededor de $44.5 \%$ de los trabajadores ganaban un salario mínimo de 1984. Es decir, en términos de los trabajadores que ganan un salario mínimo, hay una mejoría con respecto a 1984. Esto se debió a un ligero repunte de la economía mexicana entre 2005 y 2006. Teniendo como referente el salario mínimo real, en los dos periodos iniciales de la apertura comercial las condiciones de los asalariados empeoran, pero estas mejoran para el año 2006, aunque esto último no perduró para los siguientes años.

\section{Relación entre nivel escolar y salario promedio}

Para poder tener una primera aproximación a la relación escolaridad-salario, se realizaron gráficos de barras para cada año entre el nivel de escolaridad del trabajador y el salario nominal promedio que recibía.

En el año de 1984, se puede observar en la Figura 2, que la repercusión de la escolaridad en el salario promedio de los trabajadores no es tan clara. Como cabría esperar, los salarios promedio menores se encuentran entre aquellos trabajadores sin instrucción escolarizada. La mayor escolaridad reditúa en mayores salarios promedio para trabajadores con niveles de escolaridad de primaria inconclusa, y en seguida, los que tienen instrucción primaria conclusa.

Lo anterior no se mantiene para los trabajadores con niveles de escolaridad de secundaria inconclusa, pues su salario promedio es menor al que obtienen los trabajadores con primaria conclusa. Los trabajadores con nivel de escolaridad de secundaria conclusa, tienen un salario promedio superior al de los trabajadores anteriores. Sin embargo, de nueva cuenta, los trabajadores con niveles de escolaridad de pre-universidad inconclusa tienen un salario promedio menor al de los trabajadores con secundaria terminada. Al parecer para este año y para estos niveles de escolaridad, 
el mercado laboral castiga a los trabajadores con estudios truncos. Los niveles desde pre-universidad conclusa hasta posgrado tienen el comportamiento que se presume debería darse, es decir, a mayor nivel escolar, mayor salario promedio.

Figura 2. Escolaridad-Salario Promedio, 1984

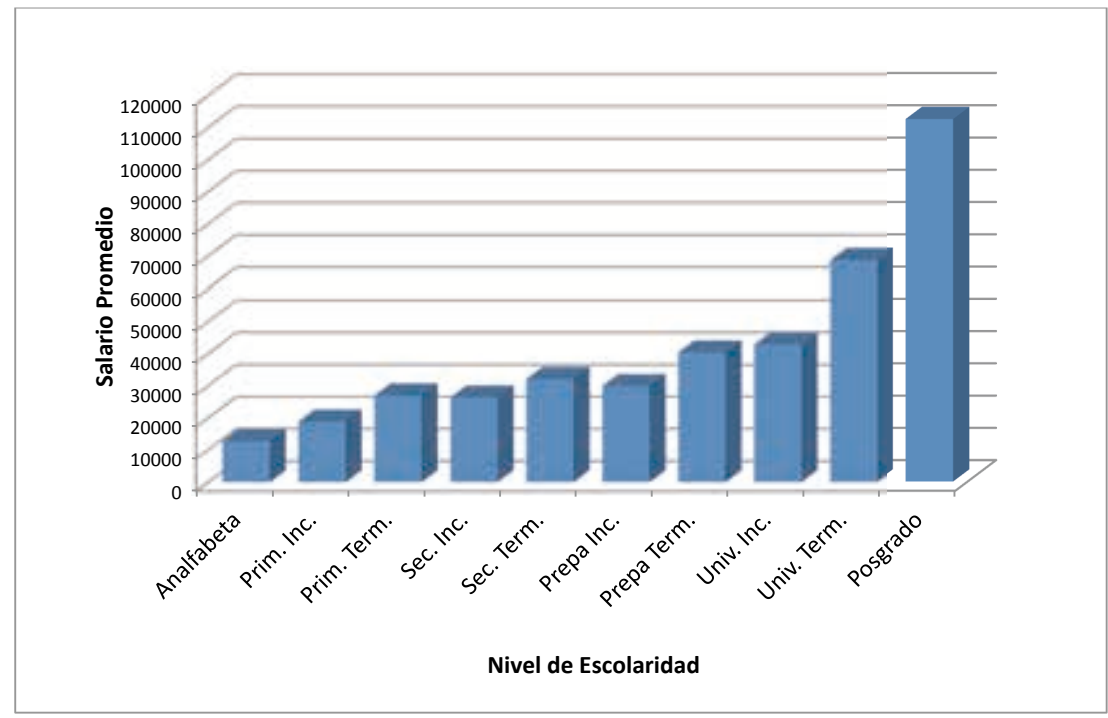

Fuente: Autor con datos de la ENIGH de 1984 (se debe tomar en cuenta el tratamiento que antes señalamos se realizó a esta encuesta).

En la Figura 2 también se puede observar, que en promedio los estudios de posgrado son bien remunerados. De los datos obtenidos, podemos decir que sólo los trabajadores sin instrucción escolarizada y con primaria inconclusa, ganaban en promedio menos de un salario mínimo nominal y que los trabajadores con estudios hasta de universidad inconclusa obtuvieron en promedio menor de dos salarios mínimos nominales. Por último, los salarios promedio más altos, con niveles de estudio de posgrado, eran de cuatro punto seis salarios mínimos nominales.

En la primera etapa de la apertura comercial, en el año de 1992, cuando México ya se encontraba inmerso en el GATT, la situación cambia con respecto a lo que sucedía en el año de 1984. Se podría decir sucintamente, que para este año los trabajadores ganan en promedio según el nivel escolarizado que tenían. Es decir, se cumple el hecho de que a mayor nivel de estudios mayor salario promedio percibido por el trabajador. En la Figura 3, se puede observar claramente la relación creciente entre nivel escolar y salario promedio. 
Figura 3. Escolaridad- Salario Promedio, 1992

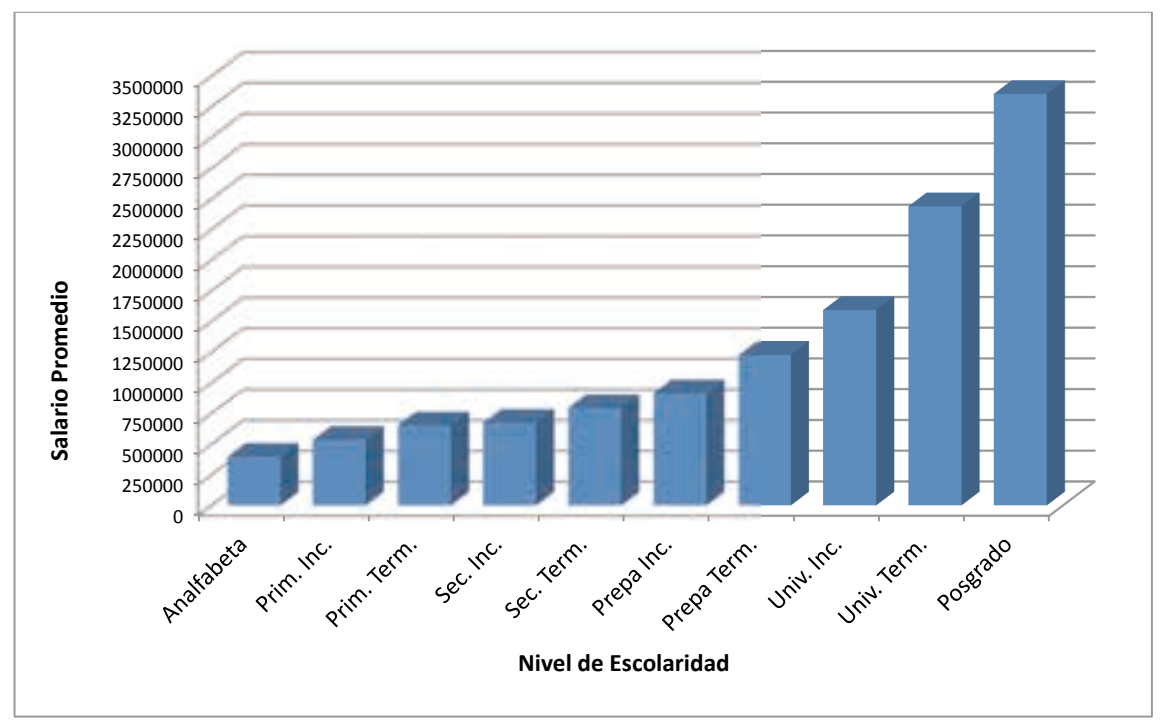

Fuente: Autor con datos de la ENIGH de 1992 (se debe tomar en cuenta el tratamiento que antes señalamos se realizó a esta encuesta).

En este año, el salario mínimo nominal sólo lo ganan en promedio los trabajadores sin ningún nivel de instrucción escolarizada. Menos de dos salarios mínimos nominales, en promedio lo ganarían los trabajadores con instrucción de secundaria terminada o menos. Con menos de tres salarios mínimos nominales, en promedio sólo agregaríamos a los trabajadores con niveles de instrucción de pre-universidad inconclusa. Los trabajadores con niveles de escolaridad de pre-universidad terminada y más, ganan en promedio más de tres salarios mínimos nominales. Por su parte, los trabajadores con niveles escolares de posgrado, ahora ganan en promedio ocho punto cuatro salarios mínimos nominales. En esta primera etapa de la apertura comercial, hay una relación directa entre nivel de escolaridad y el salario promedio.

Para el año 2000, aparentemente tenemos una relación creciente entre nivel de escolaridad y salario promedio, que se puede observar en la Figura 4. Decimos aparente, pues vuelve a ocurrir algo similar a lo que pasaba en 1984, pero en esta ocasión sólo en una situación. El salario promedio más bajo como en las anteriores ocasiones, es el de trabajadores sin escolaridad, y de ahí aumenta hasta los trabajadores con certificado de primaria. El problema aparece con los trabajadores con escolaridad de secundaria inconclusa, pues sus salarios promedio son menores a los de trabajadores con certificado de primaria. A partir de los trabajadores con certificado de estudios de secundaria y mayores, la relación creciente se retoma. 
Pero es interesante observar que para este año, en promedio, ninguno de los trabajadores con escolaridad o sin ella ganaba el sueldo mínimo nominal. Menos de dos salarios mínimos nominales, en promedio, lo ganaban los trabajadores con estudios de secundaria inconclusa o menos. Menos de tres salarios mínimos nominales, trabajadores con estudios de pre-universidad inconclusa o menos. Para este año los trabajadores con estudios de posgrado ganaban en promedio diez punto siete salarios mínimos nominales. Los trabajadores con estudios profesionales terminados ganaban en promedio seis punto dos salarios mínimos.

Figura 4. Escolaridad-Salario Promedio, 2000

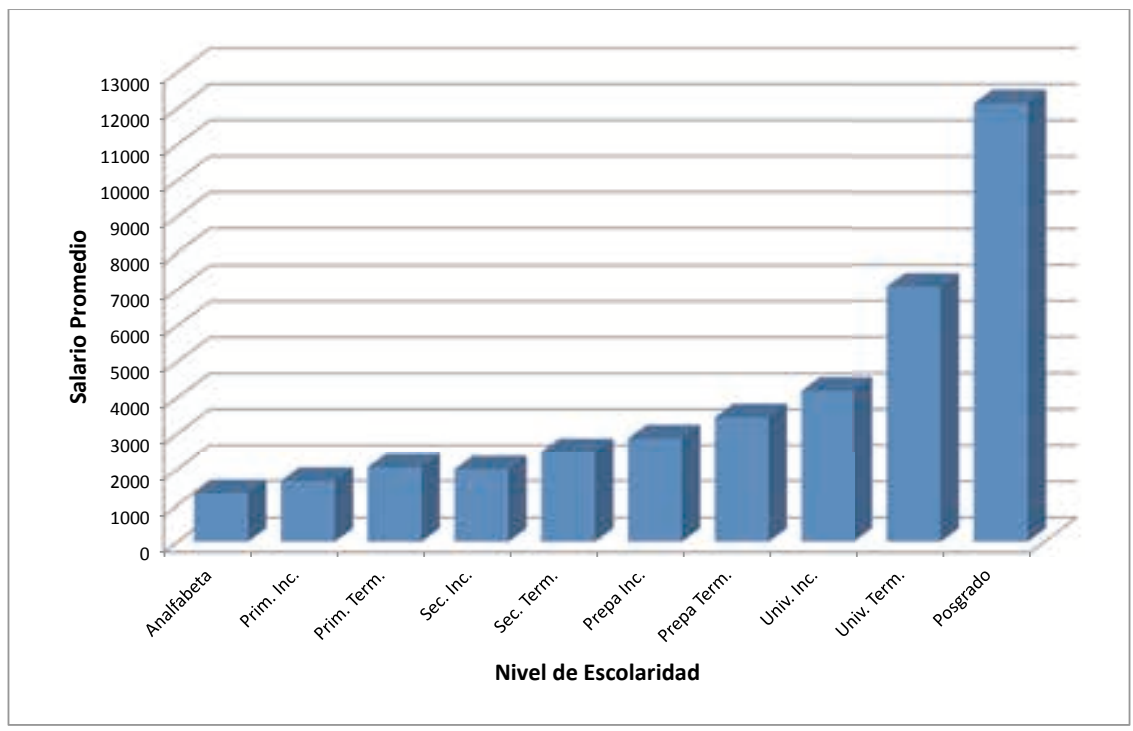

Fuente: Autor con datos de la ENIGH de 2000 (se debe tomar en cuenta el tratamiento que antes señalamos se realizó a esta encuesta).

Para el año 2006 se regresa a una situación similar a la que ocurrió en 1992. Es decir, hay una relación creciente entre el nivel escolar del trabajador y el salario promedio. En este año, se vuelve a observar que en promedio ninguno de los trabajadores con escolaridad o sin ella ganan en promedio el salario mínimo nominal. Ahora, los trabajadores que ganan menos de dos salarios mínimos nominales en promedio, son los trabajadores sin estudios y los trabajadores con estudios de primaria inconclusa. Menos de tres salarios mínimos nominales, trabajadores con estudios de secundaria inconclusa o menos. Para este año, los trabajadores con estudios de posgrado ganaban 
en promedio catorce punto nueve salarios mínimos. Los trabajadores con estudios profesionales terminados ganaban en promedio ocho punto tres salarios mínimos.

Figura 5. Escolaridad-Salario Promedio, 2006

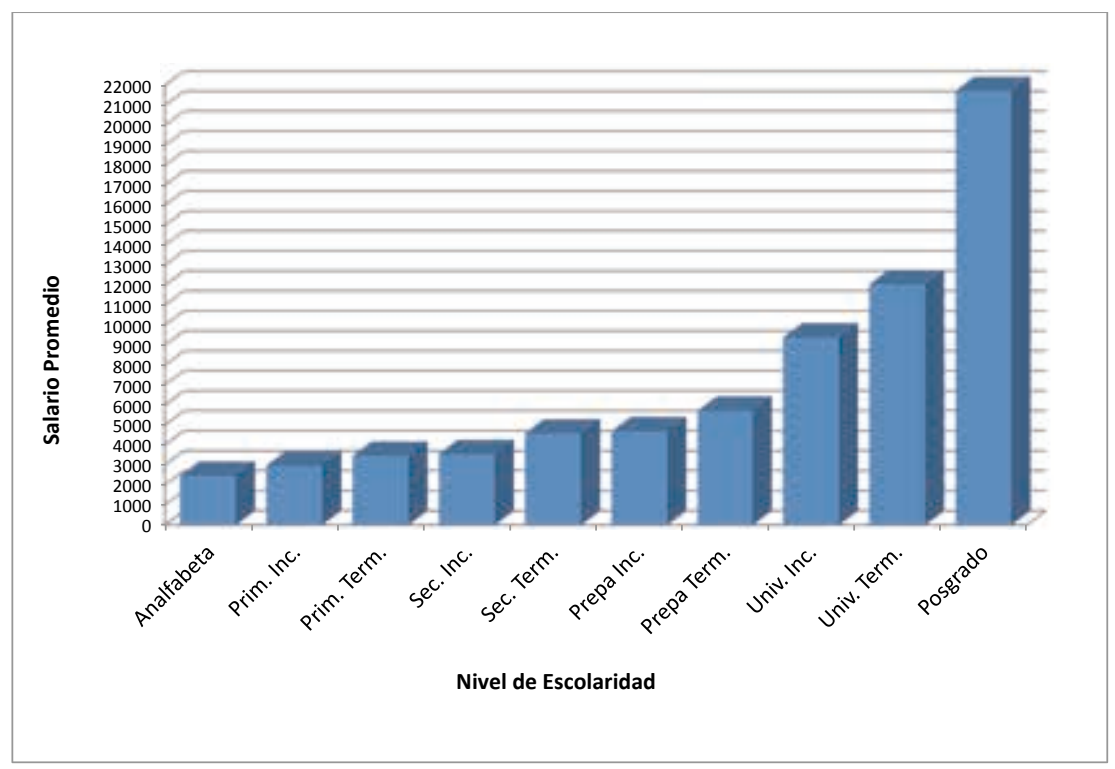

Fuente: Autor con datos de la ENIGH de 2006 (se debe tomar en cuenta el tratamiento que antes señalamos se realizó a esta encuesta).

Para este año, los trabajadores con estudios de posgrado en promedio ganan más de lo que ganaban en términos reales en los años 2000 y 1992. El aumento real promedio es considerable con respecto al que existía en el año de 1984.

Es interesante observar, cómo la relación que se esperaba en la apertura comercial, dados los mayores niveles de competencia, se da entre la escolaridad y los salarios nominales promedio. Es decir, en la apertura comercial hay una correspondencia directa creciente entre el nivel de escolaridad alcanzado por el trabajador y el salario nominal promedio que obtiene. Pero considerando el apartado anterior y el presente, y tomando como referencia los salarios mínimos reales y el año 1984, esa relación no se mantiene. En el apartado anterior se encontró que los trabajadores cada vez obtenían más salarios mínimos, pero también que el poder adquisitivo de estos ha ido a la baja desde los años ochenta ya que desde esos años, el salario mínimo real no dejó de decrecer hasta el año 2006. Ahora bien, haciendo un poco de análisis de datos de los dos apartados, se puede establecer que en promedio, son los trabajadores 
con menores niveles de escolaridad los que en términos reales han salido más perjudicados en la apertura comercial, pues su poder adquisitivo no ha dejado de caer. Por el contrario, en promedio, encontramos que los asalariados con niveles escolares superiores, en términos reales, han podido contrarrestar la pérdida de su poder adquisitivo, pues la cantidad de salarios mínimos que están ganando, ha estado aumentando en la apertura, de tal forma que han resarcido y hasta incrementado su poder adquisitivo.

\section{Desigualdad salarial por nivel de estudios alcanzado}

Para tener una primera idea del grado de desigualdad salarial que existía en las diferentes etapas analizadas, se obtuvieron los índices de Gini para la totalidad de los trabajadores en cada año. En el año de 1984, el índice de Gini obtenido es de 0.3986; para el año de 1992, es de 0.4385; para el año 2000 es de 0.4444 y para el año 2006, es de 0.49. Dado que el índice es una medida de desigualdad, podemos señalar, en una primera instancia, que la desigualdad en la distribución de los salarios ha aumentado en la apertura comercial.

Teniendo en cuenta lo anterior, se profundiza en el análisis, obteniendo los niveles de desigualdad salarial en cada uno de los niveles escolares tipificados en esta investigación y para cada uno de los años de estudio, mediante el mismo índice de Gini. Para poder observar cómo cambiaron los índices de desigualdad por nivel educativo en la apertura comercial y a que niveles educativos ha afectado más este fenómeno.

Haciendo el procedimiento antes señalado, se encontró que para el año de 1984, el mayor índice de desigualdad 0.39 estaba entre los trabajadores sin instrucción escolarizada, seguido de 0.35 entre los trabajadores con estudios de primaria inconclusos, una situación que esperábamos por la diversidad de ocupaciones en las que se pueden emplear este tipo de trabajadores. La menor desigualdad la encontramos entre los trabajadores con estudios a nivel posgrado 0.19 , una situación que también era de esperarse, pues son trabajadores muy especializados y que generalmente ocupan puestos semejantes por profesión y especialidad, por lo que se podría suponer que sus salarios no varían en gran cuantía. En seguida encontramos a los trabajadores con certificado de estudios de pre-universidad con 0.25 . Los índices para los otros niveles escolares se encuentran entre 0.31 y 0.33 , como se muestra en la Figura 6. 
Figura 6. Desigualdad, 1984

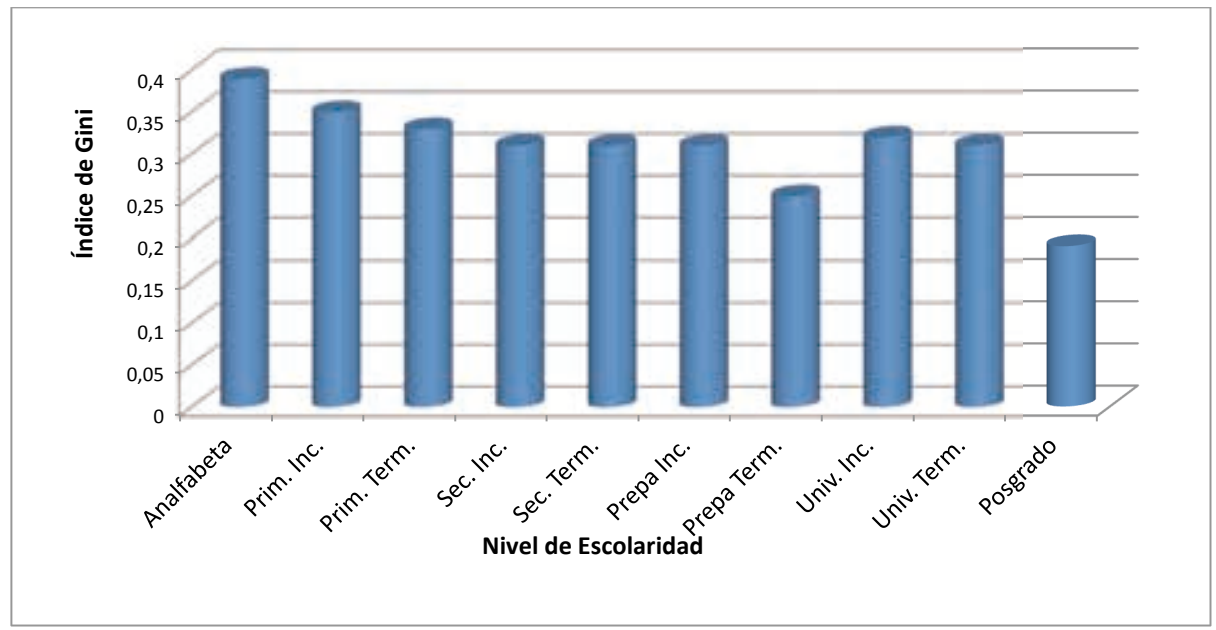

Fuente: Autor con datos de la ENIGH de 1984 (se debe tomar en cuenta el tratamiento que antes señalamos se realizó a esta encuesta).

A grandes rasgos, la situación de desigualdad entre niveles escolares tiene la lógica que podríamos esperar. Los mayores niveles de desigualdad los encontramos entre los trabajadores con menores niveles de escolaridad o sin ninguna instrucción escolarizada y los menores niveles de desigualdad entre los trabajadores con los mayores niveles de instrucción escolar.

Para la primera etapa de la apertura comercial, el anterior contexto cambia, puesto que en el año de 1984, el mayor índice de desigualdad lo encontrábamos en los trabajadores sin escolaridad y para este año se encuentra un índice de 0.40 entre los trabajadores con escolaridad universitaria complet, un nivel educativo en el que esperaríamos una baja desigualdad. También es sorpresivo que en segundo lugar estén los trabajadores con escolaridad universitaria incompleta con un índice de 0.39, y hasta tercer lugar aparezcan los trabajadores sin escolaridad con 0.38. Como se puede apreciar el índice para los trabajadores sin escolaridad, no cambia en mucho y para trabajadores con escolaridad universitaria completa, la variación es muy significativa. También cabe destacar que el índice de desigualdad aumenta drásticamente entre los trabajadores con estudios de posgrado, pues pasan de un índice de 0.19 a uno de 0.34 , un cambio muy significativo que afecta a este segmento de trabajadores asalariados. 
Cabe destacar que el nivel de desigualdad para este año, en general, se eleva, pues el índice menor en 1984 era de 0.19 y para este año el índice menor es de 0.34 . En la primera etapa de la apertura comercial, se acentúa la desigualdad entre los trabajadores asalariados que contaban con algún año en el sistema escolarizado, como se puede observar en la Figura 7.

Figura 7. Desigualdad, 1992

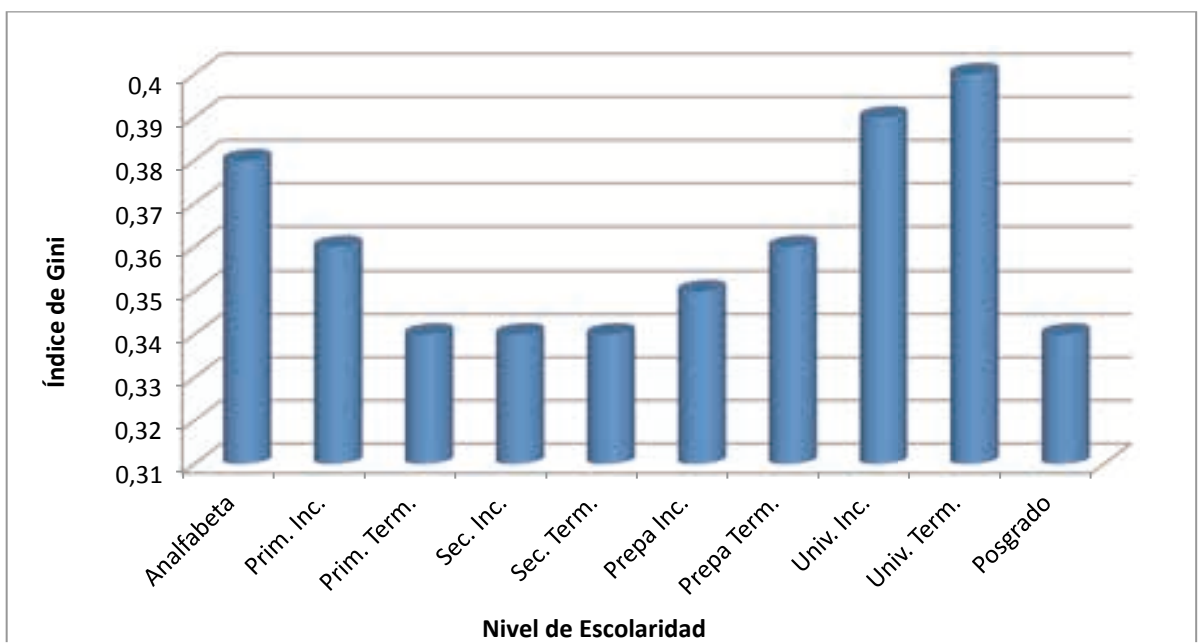

Fuente: Autor con datos de la ENIGH de 1992 (se debe tomar en cuenta el tratamiento que antes señalamos se realizó a esta encuesta).

Para la segunda etapa de la apertura comercial, algunos de los índices de desigualdad continúan aumentando, con excepción de los trabajadores sin escolaridad, los que tienen la secundaria terminada y los que tienen superior completa en las que sus índices bajan. Para esta etapa, lo más notorio es la tendencia creciente que tiene el índice de los trabajadores asalariados que tienen estudios de posgrado. Pasan de tener un índice de 0.19 en 1984 a tener un índice de 0.39 en el año 2000. La desigualdad en este segmento de trabajadores con este tipo de estudios sigue en aumento. Por otra parte, también es notorio el aumento del índice de desigualdad en trabajadores con pre-universidad completa e incompleta, como se muestra en la Figura 8. 
Figura 8. Desigualdad, 2000

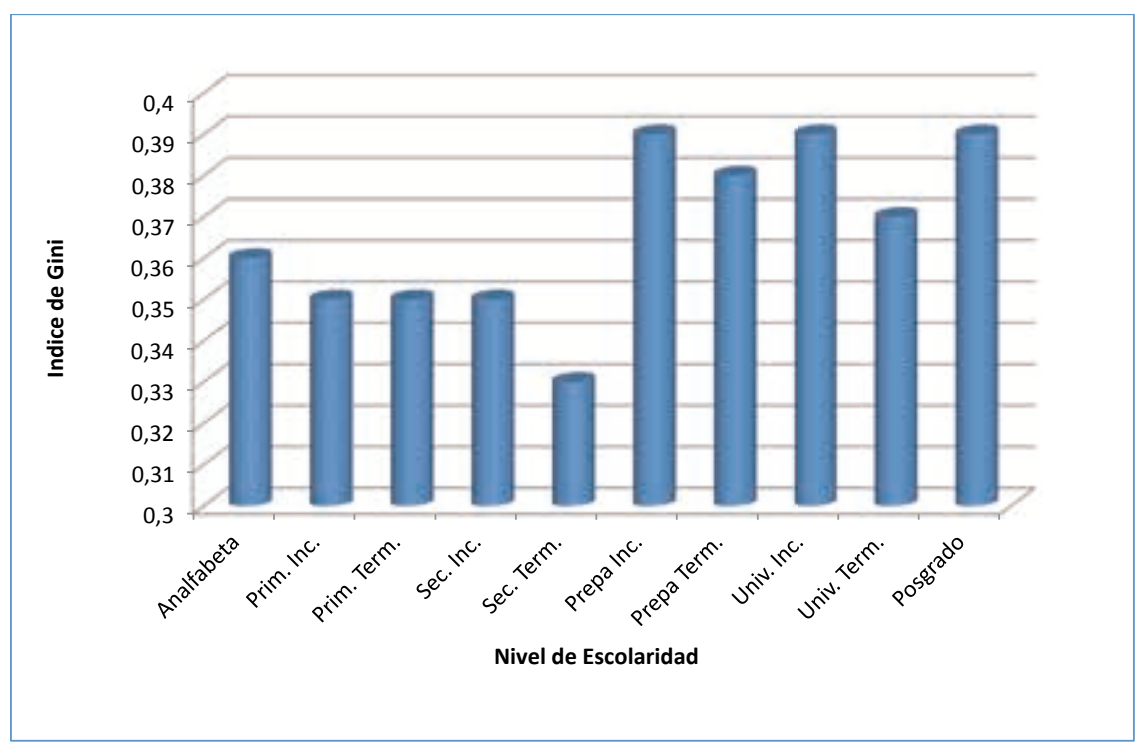

Fuente: Autor con datos de la ENIGH de 2000 (se debe tomar en cuenta el tratamiento que antes señalamos se realizó a esta encuesta).

Por último, en la tercera etapa de la apertura comercial la desigualdad prosigue aumentando, como podemos observar en la Figura 9. Ahora, el menor índice de desigualdad (0.36), lo encontramos en trabajadores con estudios de primaria terminada, pero es un índice mayor al que encontrábamos en los años anteriores. El mayor índice de desigualdad se ubica en 0.47, un índice muy superior al que encontrábamos en cualquiera de los anteriores años estudiados y que es para los trabajadores con estudios universitarios inconclusos. El siguiente mayor índice (0.45), y que de igual manera es superior a cualquiera de los otros años, es para los trabajadores con estudios de preparatoria incompleta. El tercer mayor índice de desigualdad (0.43), y que sigue siendo superior a cualquiera de los encontrados en los años analizados, es para los trabajadores con estudios de posgrado. Es sorprendente el aumento que ha tenido este índice, recordando que en 1984, era tan sólo de 0.19. El cuarto mayor índice de desigualdad (0.42), y que sigue siendo superior a cualquiera de los encontrados en los otros años, es para trabajadores con estudios de secundaria inconclusa, preparatoria terminada y universidad terminada. El siguiente mayor índice de desigualdad (0.40), y que es similar al mayor encontrado en los años anteriores, es para los trabajadores sin estudios y con estudios de secundaria. El siguiente índice (0.37) es para trabajadores con estudios de primaria inconclusa. 
Figura 9. Desigualdad, 2006

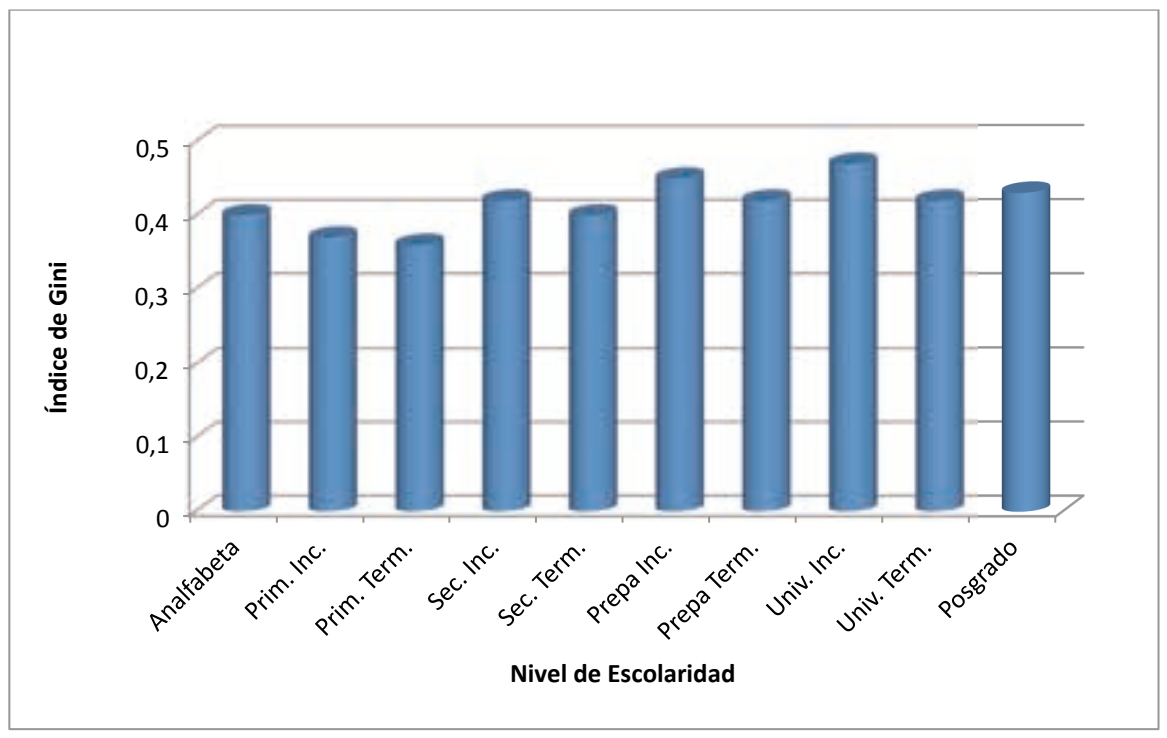

Fuente: Autor con datos de la ENIGH de 2006 (se debe tomar en cuenta el tratamiento que antes señalamos se realizó a esta encuesta).

Los índices de desigualdad, en general, se acrecientan en la apertura comercial. En un inicio esperábamos que los trabajadores con mayor escolaridad (superior y posgrado), obtendrían salarios muy semejantes o con una alteración no excesiva, dado que se suponía que sus "skills" aunque podrían variar, no lo harían de manera muy diferenciada, como se podría esperar en los trabajadores no especializados (trabajadores sin escolaridad o con niveles de primaria o secundaria), donde la desigualdad salarial sería mayor, dada la variedad de ocupaciones u oficios en las que se pueden emplear este tipo de trabajadores y la fácil y no costosa capacitación en la mayoría de ellos, tal variedad provocaría alta volatilidad en sus salarios. El panorama antes descrito, lo encontramos, en general, antes de que se diera la apertura comercial (1984), pero en la apertura comercial esto cambia, los niveles de desigualdad se van incrementando en todos los niveles escolares.

Resulta interesante resaltar, que la desigualdad ha aumentado de forma sorprendente entre los trabajadores con niveles de escolaridad de posgrado y casi se ha mantenido sin cambio entre los trabajadores sin escolaridad. Aunque, el principal énfasis se debe poner en el hecho, de que la desigualdad está aumentando en todos los niveles escolares, y que esto, simple y sencillamente nos indica que hay trabajadores con el mismo nivel escolar que están obteniendo salarios muy disímiles, por lo que, en estos casos hay otros factores que están haciendo la diferencia. 


\section{Algunas conclusiones}

Con la apertura comercial, principalmente con el NAFTA, se pensaba que los beneficios laborales iban a recaer entre los trabajadores menos calificados o con menores niveles escolares pues era una ventaja comparativa de México con sus socios (los Estados Unidos de Norteamérica y Canadá). Por el contrario, lo que encontramos en esta investigación, y que han encontrado otras investigaciones, es que los asalariados con mayores niveles escolares, han sido en promedio, los más beneficiados. Por tanto, la reflexión ante ello, es que sería rentable estudiar, pero sólo en el caso de que se lograra acceder a estudios universitarios o mayores, pues son estos trabajadores los que han podido defender o aumentar sus salarios reales (poder adquisitivo).

Pero el razonamiento no se sostiene al tener en cuenta la desigualdad salarial al interior de cada nivel educativo. Es decir, las desigualdades salariales se están incrementando entre asalariados con similar nivel escolar, lo que nos puede hablar de una calidad dispar en la educación o que otros factores más allá del nivel escolar están afectando cada vez más los salarios de los trabajadores y las bondades que se le atribuyen a la educación como factor de movilidad social. Se estaría generando así, una desigualdad que no crea oportunidades para una buena parte de la población asalariada en el presente y en un futuro para los descendientes de estos. Las oportunidades serían para un grupo muy reducido de la población. Aquel grupo que en el presente tienen los salarios necesarios y puede hacer que sus hijos accedan a niveles educativos superiores, pero que además buscan una educación de calidad como otro factor que facilita la inserción de estos al mercado que podemos llamar de los buenos trabajos y salarios altos. Además, que por los niveles educativos y los puestos laborales en que se desempeñan pueden tener acceso a información sobre las condiciones del mercado de trabajo o sobre las vacantes en puestos clave o en su caso pueden recomendar a sus hijos o enchufarlos en algún buena plaza laboral.

Por tanto, el mayor grupo de asalariados, que no cuenta con estos otros factores o que por alguna situación puede acceder a uno o algunos de ellos y no tiene tan claras sus oportunidades en el mercado laboral, están viendo a la desigualdad como un mal social que les niega la movilidad y los sentencia a perdurar por generaciones en las mismas o peores condiciones salariales, lo que algunos investigadores llaman trampas de pobreza, pues acceder a los otros factores tiene un costo y es por ello que pocos pueden acceder a ellos.

Se está produciendo una creciente desigualdad que se está tornando destructiva. Una que pensamos crea resentimiento social, entre las capas más desfavorecidas de la población, por la falta de oportunidades y movilidad social. La neutralidad distributiva del mercado y la desigualdad que crea oportunidades, están perdiendo la batalla y son otros factores los que están influyendo en mayor medida en el salario de los trabajadores. 


\section{Referencias}

Aguirre, M. (1993). Evolución del salario mínimo diario en México de 1970 a 2006. Disponible en: http://mexicomaxico.org/Voto/SalMinInf.htm

Berman, E., Bound, J. y Griliches, Z. (1994). Changes in the demand for skiller labour within U.S. manufacturing: Evidence from the Annual Survey of Manufacturers. The Quarterly Journal of Economics, 109 (2), 367-397.

Blackburn, M., Bloom, D. and Freeman, R. (1990/91). An Era of Falling Earning andRising Inequality. Brookings Review, winter.

Bluestone, B. y Harrison, B. (1988). The great U-Turn: Corporate restructuring and polarization of America, New York, USA: Basic Books.

Castro, D. y Huesca, L. (2005). Salarios y desigualdad territorial en las áreas urbanas de México 1992-2002. Tesis Doctoral, Universidad Autónoma de Barcelona.

Castro, D. y Huesca, L. (2007). Desigualdad salarial en México: Una Revisión. Papeles de Población, octubre-diciembre, (54), 225-264. México: Universidad Autónoma del Estado de México.

Cragg, M.I. y Epelbaum, M. (1996). Why has wage dispersion grow in Mexico? Is it the incidence of reforms or the growing demand for skills? Journal of Development Economics, 51(1), 99-116.

Davis, S.J. y Haltiwanger, J. (1991). Wage dispersion between and within U.S. manufacturing plants, 1963-86. Brookings Papers on Economic Activity. Microeconomics, 1991, 115-200.

Feliciano, Z. (2001). Workers and trade liberalization: The impact of trade reforms in Mexico on wages and employment. Industrial and Labor Relations Review, 55 (1), 95-115.

Guillén, H. (1988). De la crisis financiera a la austeridad hayekiana en México. México: Siglo XXI.

Hanson, G.H. (2005). Globalization, labor income and poverty in Mexico. NBER Working Papers, 11027. Cambridge, MA: National Bureau Economic Research.

Hanson, G.H. (2003). What has happened to wages in Mexico since NAFTA? Implications for Hemispheric Free Trade. NBER Working Papers, 9563. Cambridge, MA: National Bureau Economic Research.

Hanson, G. y Harrison, A. (1999). Trade liberalization and Wage Inequality in Mexico. Industrial and Labour Relation Review, 52 (2), 271-288 
Hanson, G. y Harrison, A. (1995). Trade technology and wage inequality. NBER Working Papers, 5110. Cambridge, MA: National Bureau Economic Research.

Herrera, F. y Melgoza J. (2003). Evolución reciente de la filiación sindical y la regulación laboral, Enrique de la Garza y Carlos Salas (coords.). La situación del trabajo en México, AFL/CIO/UNAM/Instituto de Estudios del Trabajo y Plaza Valdés.

Instituto Nacional de Estadística y Geografía. (1984). Encuesta Nacional de Ingresos y Gastos de los Hogares (ENIGH). [CD-ROM] México: INEGI.

Instituto Nacional de Estadística y Geografía. (1992). Encuesta Nacional de Ingresos y Gastos de los Hogares (ENIGH). [CD-ROM] México: INEGI.

Instituto Nacional de Estadística y Geografía. (2000). Encuesta Nacional de Ingresos y Gastos de los Hogares (ENIGH). [CD-ROM] México: INEGI.

Instituto Nacional de Estadística y Geografía. (2006). Encuesta Nacional de Ingresos y Gastos de los Hogares (ENIGH). [CD-ROM] México: INEGI.

Instituto Nacional de Estadística y Geografía (1998) Estadística de la Industria Maquiladora de Exportación (1980-1993).

Juhn, C., Murphy, K. y Pierce, B. (1993). Wage inequality and the rise in returns to skill. Economy, 101 (3), 410-441.

Kosters, M. H. (1990). Schooling, work experience and wage trends. The American Economic Review, 80 (2), 308-312.

Krueger, A.B. (1993). How computers have changed the wage structure? Evidence from Microdata, 1984-1989. The Quarterly of Journal of Economics, 108 (1), 33 60 .

Lawrence, R.Z. y Slaughter, M.J. (1993). International trade and american wages in the 1980s: giant sucking sound or small hiccup? Brooking Papers on Economic Activity. Microeconomics, 1993 (2), 161-210.

López, G. (2001). Evolution of earnings and rate of returns to education in Mexico, en Working Paper, the World Bank.

López, G. (2004). Mexico: evolution of earnings, inequality and rates of returns to education (1988-2002), Earnings Inequality Study at the World Bank Report (1994), the World Bank, 211-263.

Luyando, J. R. (2012). Posible segmentación institucional del mercado laboral mexicano. V Jornadas de Economía Crítica. Buenos Aires: Universidad de Buenos Aires. 
Luyando, J. R. (2011). Microeconomía de un mercado de trabajo dual: una reconsideración al modelo de Salop. Trayectorias, (32), 71-93.

Meza, L. (1999). Cambios en la estructura salarial de México en el periodo 1988-1993 y el aumento en el rendimiento de la educación superior. El Trimestre Económico, (262).

Murphy, K.M. y Welch, F. (1991). Wage premiums for college graduates: Recent growth and possible explanations. Educational Researcher, 18(4), 17-26.

Popli, Gurleen K. (2006). Rising wage inequality in Mexico, 1984-2000: a distributional analysis. Munich Personal RePEc Archive (MPRA).

Popli, Gurleen K. (2005). Rising wage inequality in Mexico: structural reforms or changing labor market institutions. Sheffield economic research paper series (2005016).

Rodríguez, A. y Sánchez J. (2002). Economic polarisation through trade: the impact of trade liberalization on mexico's regional growth. Papaer presented at the Cornell/ LSE/Winder Conference on Spatial Inequality and Development, London School of Economics, June 1-43.

Slaughter, M. (1999). Globalisation and wage: A tale of two perspectives. The World Economy, 22(5), 609-629.

Stiglitz, J. (2002). El malestar en la globalización. México: Taurus.

Welch, Finis (1999). In Defense of Inequality. The American Economic Review, 89 (2), May, 1-17. 